\title{
On contraction and the modal fragment
}

\author{
Kai Brünnler Dieter Probst Thomas Studer
}

October 23, 2007

\begin{abstract}
We observe that removing contraction from a standard sequent calculus for first-order predicate logic preserves completeness for the modal fragment.
\end{abstract}

\section{Introduction}

From a proof-theoretic perspective, one could say that the rule of contraction is the reason for the undecidability of first-order logic. Contraction basically states that whenever we have derived a premise $A \vee A$, then we are allowed to infer $A$. Such a structural rule leads to undecidability since in a proof search procedure the following may happen: Assume we have to show that a formula $A$ holds. This formula may have been derived by the rule of contraction. Thus we have to show that $A \vee A$ holds. Again this formula may have been derived by contraction. Now we have to show that $(A \vee A) \vee(A \vee A)$ holds and so on ad infinitum.

If contraction is excluded from a deductive system for first-order logic (or only a restricted form of it is available), then no such infinite paths are possible in the proof search procedure and thus it becomes decidable whether a formula is derivable or not. However, there is no syntactic characterization available for the derivable formulae of a contraction free fragment of first-order logic. Ketonen and Weyhrauch [6] investigate a deductive system without contraction. They can show that for each valid formula, there is a logically equivalent formula which can be derived in their calculus. Grishin [5] also studies the contraction free fragment. He presents an algebraic semantics for which he can prove completeness. Moreover, he introduces a set theory that is based on contraction-free logic. Cantini [2] further investigates Grishin's set theory and shows an undecidability result. A related system is studied by Petersen [7]. 
We present a one-sided sequent calculus $T$ in which only a controlled form of contraction is available. T derives sequents, that are finite multisets of formulae which are interpreted disjunctively. Usually contraction is built into deductive systems either by adding it explicitly as structural rule or by formulating the logical rules such that they contain contraction implicitly (this concerns in particular the rule for existential quantification). In T neither do we have a rule for contraction nor is contraction incorporated into the rule for existential quantification. Hence, $T$ includes the following rule for existential quantification

$$
\frac{\Phi, B(u)}{\Phi, \exists u B(u)} \quad(\exists c)
$$

Thus, from the premise $\exists u A(u), A(u)$ we can infer $\exists u A(u), \exists u A(u)$ but not $\exists u A(u)$.

The only place where a (weak) form of contraction occurs in $\mathrm{T}$ is the rule for conjunctions

$$
\frac{\Phi, A \quad \Phi, B}{\Phi, A \wedge B}(\wedge)
$$

In this rule, $\Phi$ occurs twice in the premises but only once in the conclusion. Thus there is some contraction built in, but this does not make $T$ undecidable.

In the present note, we prove completeness of $T$ with respect to the modal fragment of first-order logic. This fragment is given by the standard firstorder translation of multi-modal logic $\mathrm{K}_{n}$ [1]. Therefore, we can make use of a contraction-free deductive system for $\mathrm{K}_{n}$ to establish completeness of $\mathrm{T}$ for the modal fragment. Hence, we obtain a syntactic characterization of a fragment of first-order logic for which $\mathrm{T}$ is complete.

\section{First-Order Logic}

We work with the language $\mathcal{L}_{1}$ of first-order logic which comprises countably many free and bound variables, countably many relation symbols of every arity $\left(P_{1}, P_{2}, \ldots\right.$ are the unary relation symbols and $R_{1}, R_{2}, \ldots$ are the binary relation symbols of $\mathcal{L}_{1}$ ) as well as the logical symbols $\sim$ (atomic negation), $\vee$ (disjunction), $\wedge$ (conjunction), $\exists$ and $\forall$ (existential and universal quantification). We let $u, v, w$ range over variables. The notation $A(u)$ is used to indicate that the variable $u$ may occur free in $A$. By $\mathrm{FV}(A)$ we denote the set of variables occurring in free in $A$. The modal fragment $\mathcal{L}_{1}^{M}$ is a subset of the formulae of $\mathcal{L}_{1}$ as defined below.

Definition 1. The formulae of $\mathcal{L}_{1}^{M}$ are defined inductively as follows. 
1. If $P$ is a unary relation symbol, then $P(u)$ and its negation $\sim P(u)$ are (atomic) $\mathcal{L}_{1}^{M}$ formulae for every variable $u$.

2. If $A$ and $B$ are $\mathcal{L}_{1}^{M}$ formulae with $\mathrm{FV}(A)=\mathrm{FV}(B)$ then $A \wedge B$ and $A \vee B$ are $\mathcal{L}_{1}^{M}$ formulae.

3. Let $R$ be a binary relation symbol and $B(v)$ be an $\mathcal{L}_{1}^{M}$ formula. Then

$$
\forall v(\sim R(u, v) \vee B(v)) \text { and } \exists v(R(u, v) \wedge B(v))
$$

are $\mathcal{L}_{1}^{M}$ formulae for every variable $u$ which is different from $v$.

Note that an $\mathcal{L}_{1}^{M}$ formula contains exactly one variable free.

We will use the standard semantics for first-order languages. If $\mathcal{M}$ is an interpretation and $A$ is a formula, then $\mathcal{M} \models A$ means that $A$ holds in $\mathcal{M}$. A formula $A$ is valid, denoted by $\models A$, if it holds in all interpretations.

Next, we are going to present the one-sided sequent calculus $T$ in which only a restricted form of contraction is available. A sequent is a finite multiset of formulae. We employ $\Gamma, \Delta, \Sigma, \Phi, \ldots$ to denote sequents. $T$ comprises the following axioms and rules.

Axioms: For all sequents $\Phi$ and atomic formulae $P$ of $\mathcal{L}_{1}$

$$
\Phi, P, \sim P \quad(A x) .
$$

Propositional rules: For all sequents $\Phi$ and formulae $A$ and $B$ of $\mathcal{L}_{1}$

$$
\frac{\Phi, A, B}{\Phi, A \vee B} \quad(\vee), \quad \frac{\Phi, A \quad \Phi, B}{\Phi, A \wedge B}(\wedge),
$$

Quantifier rules: For all sequents $\Phi$, formulae $B$, and variables $u$ of $\mathcal{L}_{1}$

$$
\frac{\Phi, B(u)}{\Phi, \forall u B(u)}
$$

where we assume that the variable $u$ does not occur free in the conclusion $\Phi, \forall u B(u)$, and

$$
\frac{\Phi, B(u)}{\Phi, \exists u B(u)} \quad(\exists c)
$$

By induction on the length of derivations, we can easily see that a weakening lemma holds for $\mathrm{T}$.

Lemma 2. For all sequents $\Gamma$ and $\Delta$ we have $\mathrm{T} \vdash \Gamma \Longrightarrow \mathrm{T} \vdash \Gamma, \Delta$.

Remark 3. If we replace the rule $(\exists c)$ with the following

$$
\frac{\Phi, \exists u B(u), B(u)}{\Phi, \exists u B(u)} \quad(\exists),
$$

then we obtain a system which is complete for full first-order logic. 


\section{Modal Logic}

We use the language $\mathcal{L}_{M}$ of multi-modal logic which comprises countably many atomic propositions $p_{1}, p_{2}, \ldots$ and the symbols $\sim$ (atomic negation), $\mathrm{V}$ (disjunction), $\wedge$ (conjunction), $\diamond_{i}$ and $\square_{i}$ (modal operators) for every natural number $i$.

We make use of the standard Kripke semantics for modal languages. A formula of $\mathcal{L}_{M}$ is valid if it is satisfied in every world in every Kripke structure. The deductive system $\mathrm{K}$ for multi-modal logic derives finite multisets (sequents) of $\mathcal{L}_{M}$ formulae. Again, only the $(\wedge)$ rule has a restricted form of contraction built-in. The system $\mathrm{K}$ is defined by the following axioms and rules:

Axioms: For all sequents $\Gamma$ and propositions $p$ of $\mathcal{L}_{M}$

$$
\Gamma, p, \sim p \quad(A x) .
$$

Propositional rules: For all sequents $\Gamma$ and formulae $A$ and $B$ of $\mathcal{L}_{M}$

$$
\frac{\Gamma, A, B}{\Gamma, A \vee B} \quad(\vee), \quad \frac{\Gamma, A \quad \Gamma, B}{\Gamma, A \wedge B}(\wedge) .
$$

Modal rules: For all sequents $\Gamma$ and $\Sigma$ and formulae $A$ of $\mathcal{L}_{M}$ and all natural numbers $i$

$$
\frac{\Gamma, A}{\diamond_{i} \Gamma, \square_{i} A, \Sigma}
$$

where $\diamond_{i}\left\{B_{1}, \ldots, B_{k}\right\}:=\left\{\diamond_{i} B_{1}, \ldots, \diamond_{i} B_{k}\right\}$.

Soundness and completeness of $\mathrm{K}$ can be obtained by standard methods, see for instance $[3,4]$. So we will only sketch a completeness proof.

Theorem 4. The system $\mathrm{K}$ is sound and complete for $\mathcal{L}_{M}$ formulae.

Proof. We call a finite set $\Phi$ of $\mathcal{L}_{M}$ formulae saturated if

1. $\mathrm{K} \nvdash \Phi$,

2. $A \wedge B \in \Phi$ implies $A \in \Phi$ or $B \in \Phi$, and

3. $A \vee B \in \Phi$ implies $A \in \Phi$ and $B \in \Phi$.

It is easy to show that

for each sequent $\Delta$ with $\mathrm{K} \nvdash \Delta$, there exists a saturated set $\Phi$

such that $\Phi$ is a superset of the underlying set of $\Delta$.

We define the Kripke structure $\mathcal{M}=\left(W, R_{1}, \ldots, R_{n}, \lambda\right)$ as follows: 
1. $W$ consists of all saturated sets,

2. for any $\Phi, \Psi \in W$ we set $(\Phi, \Psi) \in R_{i}$ if $\left\{A: \diamond_{i} A \in \Phi\right\} \subseteq \Psi$,

3. $\lambda(p):=\{\Phi \in W: p \notin \Phi\}$.

By induction on the structure of the formula $A$ we can show that

for all formulae $A$ and all $\Phi \in W$ we have $A \in \Phi \Rightarrow \mathcal{M}, \Phi \not \forall A$.

We only show the case for $A=\square_{i} B$. We have $\mathrm{K} \forall B,\left\{C: \diamond_{i} C \in \Phi\right\}$, since otherwise by the $(\square)$ rule we would obtain $\mathrm{K} \vdash \Phi$ which contradicts $\Phi$ saturated. By (1) there exists $\Psi$ saturated with $B,\left\{C: \diamond_{i} C \in \Phi\right\} \subseteq \Psi$. By the induction hypothesis we obtain $\mathcal{M}, \Psi \not \models B$. The definition of $R_{i}$ gives us $(\Phi, \Psi) \in R_{i}$. Hence we conclude $\mathcal{M}, \Phi \not \forall \square_{i} B$.

To obtain completeness of $\mathrm{K}$ assume $\mathrm{K} \forall \forall A$ for some formula $A$. By (1) there exits a saturated set $\Phi$ which contains $A$. By (2) we find $\mathcal{M}, \Phi \not \models A$. Thus $A$ is not valid.

\section{Completeness}

In this section, we show completeness of $\mathrm{T}$ with respect to $\mathcal{L}_{1}^{M}$ formulae. We start with recalling the inductive definition of the standard translation $\mathrm{ST}_{u}(\cdot)$.

1. $\mathrm{ST}_{u}\left([\sim] p_{i}\right):=[\sim] P_{i}(u)$,

2. $\mathrm{ST}_{u}(A * B):=\mathrm{ST}_{u}(A) * \mathrm{ST}_{u}(B)$ for $* \in\{\vee, \wedge\}$,

3. $\mathrm{ST}_{u}\left(\square_{i} A\right):=\forall v\left(\sim R_{i}(u, v) \vee \mathrm{ST}_{v}(A)\right)$,

4. $\mathrm{ST}_{u}\left(\diamond_{i} A\right):=\exists v\left(R_{i}(u, v) \wedge \mathrm{ST}_{v}(A)\right)$,

5. $\mathrm{ST}_{v}\left([\sim] p_{i}\right):=[\sim] P_{i}(v)$,

6. $\mathrm{ST}_{v}(A * B):=\mathrm{ST}_{v}(A) * \mathrm{ST}_{v}(B)$ for $* \in\{\vee, \wedge\}$,

7. $\mathrm{ST}_{v}\left(\square_{i} A\right):=\forall u\left(\sim R_{i}(v, u) \vee \mathrm{ST}_{u}(A)\right)$,

8. $\mathrm{ST}_{v}\left(\diamond_{i} A\right):=\exists u\left(R_{i}(v, u) \wedge \mathrm{ST}_{u}(A)\right)$.

where $v$ is a variable different from $u$. For a sequent $\Phi=A_{1}, \ldots, A_{n}$ of $\mathcal{L}_{M}$ formulae, we define $\mathrm{ST}_{u}(\Phi)=\mathrm{ST}_{u}\left(A_{1}\right), \ldots, \mathrm{ST}_{u}\left(A_{n}\right)$.

If we identify $\mathcal{L}_{1}^{M}$ formulae that differ only in the names of bound variables (whether an $\mathcal{L}_{1}^{M}$ formula is provable in $\mathrm{T}$ does not depend on the names of 
its bound variables), then each $\mathcal{L}_{1}^{M}$ formula $A(u)$ is the standard translation $\mathrm{ST}_{u}(C)$ of some $\mathcal{L}_{M}$ formula $C$, and conversely, for each $\mathcal{L}_{M}$ formula $C$, $\mathrm{ST}_{u}(C)$ is an $\mathcal{L}_{1}^{M}$ formula.

Lemma 5. Let $\Phi$ be a sequent of $\mathcal{L}_{M}$ formulae. Then

$$
\mathrm{K} \vdash \text { 品 } \Phi \Longrightarrow \mathrm{T} \vdash \mathrm{ST}_{u}(\Phi)
$$

for each variable $u$ of $\mathcal{L}_{1}$.

Proof. The claim is easily shown by induction on $n$. The only critical case is if $\mathrm{K} \vdash^{n} \Phi$ has been obtained by a $(\square)$ rule. Let $\Phi:=\diamond_{i} \Psi, \square_{i} A, \Xi$ and $\Gamma:=\mathrm{ST}_{u}(\Phi)$ which is then of the form

$$
\exists v\left(R_{i}(u, v) \wedge B_{1}(v)\right), \ldots, \exists v\left(R_{i}(u, v) \wedge B_{k}(v)\right), \forall v\left(\sim R_{i}(u, v) \vee C(v)\right), \Sigma .
$$

By the induction hypothesis we get $\mathrm{T} \vdash B_{1}(v), \ldots, B_{k}(v), C(v)$. Adding $\sim R_{i}(u, v)$ by weakening yields $\mathrm{T} \vdash B_{1}(v), \ldots, B_{k}(v), \sim R(u, v), C(v)$. Now consider the following derivation in $\mathrm{T}$ where $\Delta:=B_{2}(v), \ldots, B_{k}(v)$.

$$
\begin{gathered}
\frac{R_{i}(u, v), \Delta, \sim R_{i}(u, v), C(v) \quad B_{1}(v), \Delta, \sim R_{i}(u, v), C(v)}{R_{i}(u, v) \wedge B_{1}(v), \Delta, \sim R_{i}(u, v), C(v)} \\
\frac{}{\exists v\left(R_{i}(u, v) \wedge B_{1}(v)\right), \Delta, \sim R_{i}(u, v), C(v)} \\
\frac{\vdots}{\exists v\left(R_{i}(u, v) \wedge B_{1}(v)\right), \ldots, \exists v\left(R_{i}(u, v) \wedge B_{k}(v)\right), \sim R_{i}(u, v), C(v)} \\
\exists v\left(R_{i}(u, v) \wedge B_{1}(v)\right), \ldots, \exists v\left(R_{i}(u, v) \wedge B_{k}(v)\right), \sim R_{i}(u, v) \vee C(v) \\
\hline v\left(R_{i}(u, v) \wedge B_{1}(v)\right), \ldots, \exists v\left(R_{i}(u, v) \wedge B_{k}(v)\right), \forall v\left(\sim R_{i}(u, v) \vee C(v)\right)
\end{gathered}
$$

Again applying weakening yields $T \vdash \Gamma$ which finishes our proof.

Theorem 6. For each $\mathcal{L}_{1}^{M}$ formula $A(u)$,

$$
\mathrm{T} \vdash A(u) \Longleftrightarrow \models A(u)
$$

Proof. The direction from left to right is the standard soundness result. To show the direction from right to left assume that $A(u)$ is valid. There is an $\mathcal{L}_{M}$ formula $B$ such that $\mathrm{ST}_{u}(B)=A(u)$ (modulo renaming of bound variables). Thus $\mathrm{ST}_{u}(B)$ is a valid $\mathcal{L}_{1}^{M}$ formula and therefore, $B$ is valid with respect to the Kripke semantics. Completeness of $\mathrm{K}$ gives us $\mathrm{K} \vdash B$. By the above lemma, we finally conclude $\mathrm{T} \vdash A(u)$. 


\section{Conclusion}

We have introduced a first-order calculus $\mathrm{T}$ in which only a restricted form of contraction is available. Making use of a deductive system for propositional multi-modal logic, we have established completeness of $\mathrm{T}$ for the modal fragment of first-order logic. Our work provides a first step towards a syntactic characterization of first-order formulae that are derivable with a restricted form of contraction.

We believe that calculi with controlled contraction are a proof-theoretic answer to the question about the robust decidability of modal logics [8]. Thus our work complements the model-theoretic and automata-theoretic point of view on this issue. From the perspective of first-order logic, the principles underlying the decidability of modal logics have not been well understood. The old argument that the modal fragment is contained in the two variable fragment of first-order logic is too simple and does not explain the decidability of modal logic in an appropriate way. In our opinion, the approach using controlled contraction provides a better explanation. For instance, the proof search procedure in $\mathrm{T}$ immediately shows that the validity problem for $\mathrm{K}_{n}$ is in PSPACE. A result which does not follow from the two variable argument.

The modal fragment only characterizes a subset of the formulae provable without contraction. Obvious candidates for a full characterization would be the so-called guarded fragments. However the following guarded formula is valid but not derivable in such a calculus

$\sim P(u) \vee \exists x(Q(x) \wedge \forall y(\sim R(y) \vee \sim P(y))) \vee \exists x(P(x) \wedge(R(x) \vee \forall y \sim Q(y)))$

This leads to two questions.

1. Is there a syntactic characterization of formulae provable without contraction?

2. Can we characterize guarded fragments in terms of some restriction of contraction?

We hope that the observations in the present note provide a first step towards answers to these questions.

\section{References}

[1] Patrick Blackburn, Maarten de Rijke, and Yde Venema. Modal Logic. Cambridge University Press, 2001. 
[2] Andrea Cantini. The undecidability of Grisin's set theory. Studia Logica, 74(3):345-368, 2003.

[3] Melvin Fitting. Modal proof theory. In P. Blackburn et al., editor, Handbook of Modal Logic, pages 85-138. Elsevier, 2007.

[4] Rajeev Goré. Tableau methods for modal and temporal logics. In M. D'Agostino et al., editor, Handbook of Tableau Methods, pages 297396. Kluwer, 1999.

[5] V.N. Grishin. Predicate and set-theoretic calculi based on logic without contractions. Math. USSR. Izvestija, 18:41-59, 1981.

[6] Jussi Ketonen and Richard Weyhrauch. A decidable fragment of predicate calculus. Theoretical Computer Science, 32:297-307, 1984.

[7] Uwe Petersen. Logic without contraction as based on inclusion and unrestricted abstraction. Studia Logica, 64(3):365-403, 2000.

[8] Moshe Y. Vardi. Why is modal logic so robustly decidable? In Neil Immerman and Phokion G. Kolaitis, editors, Descriptive Complexity and Finite Models, pages 149-184, 1996.

\section{Address}

Kai Brünnler, Dieter Probst, Thomas Studer Institut für Informatik und angewandte Mathematik, Universität Bern Neubrückstrasse 10, CH-3012 Bern, Switzerland \{kai, probst, tstuder\}@iam. unibe.ch 